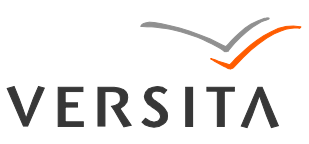

GEOCHRONOMETRIA 38(3) 2011: 223-230

DOI 10.2478/s13386-011-0033-6

Available online at

www.springerlink.com

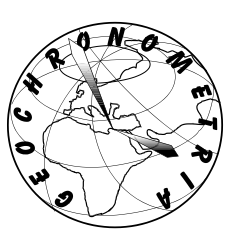

\title{
AN ATTEMPT TO CORRECT FOR THE FADING IN MILLION YEAR OLD BASALTIC ROCKS
}

\author{
P. MORTHEKAI ${ }^{1,2}$, MAYANK JAIN ${ }^{2}$, PEDRO P. CUNHA ${ }^{3}$, \\ JOSÉ M. AZEVEDO ${ }^{4}$ and ASHOK K. SINGHVI ${ }^{1}$ \\ ${ }^{I}$ Physical Research Laboratory, Navrangpura, Ahmedabad - 380 009, India \\ ${ }^{2}$ Radiation Research Division, Risoe National Laboratory for Sustainable Energy, Technical University of Denmark, \\ DK-4000 Roskilde, Denmark \\ ${ }^{3}$ IMAR-Marine and Environmental Research Centre, Department of Earth Sciences, University of Coimbra, \\ 3000-272 Coimbra, Portugal \\ ${ }^{4}$ Centre for Geophysics, Department of Earth Sciences, University of Coimbra, 3000-272 Coimbra, Portugal
}

Received 8 July 2010

Accepted 20 December 2010

\begin{abstract}
The use of feldspar for luminescence dating has been restricted because of anomalous fading. This has made its application to several important geological problems such as volcanic terrains difficult. Presently, two correction procedures are used to correct for anomalous fading. The present study tests these correction procedures using volcanic samples of known ages spanning the time period of $400 \mathrm{ka}$ to $2.2 \mathrm{Ma}$. These correction procedures provided grossly underestimated ages (up to $60 \%$ ). The possible causes for the underestimation are discussed.
\end{abstract}

Keywords: Anomalous fading, fading correction, dating of basaltic rocks.

\section{INTRODUCTION}

Anomalous fading is ubiquitous in feldspar minerals and leads to undesirable loss of trapped charge, causing an underestimation of ages. This restricts the use of feldspar as a natural dosimeter for chronological purposes both in terrestrial and in extraterrestrial settings. In the latter, often feldspar is the only luminescent phase. There have been considerable attempts to either circumvent or correct for the fading loss in nature. The first attempt was made by Huntely and Lamothe (2001) and they have demonstrated that the correction is possible for the samples below 20-50 ka. More recently a new mathematical expression was published by Huntley (2006) to describe the loss in signal due to anomalous fading after an instantaneous irradiation. Using this, Kars et al. (2008) pro-

Corresponding author: P. Morthekai

e-mail: morthekai@gmail.com posed a correction procedure that was successful in predicting the field saturation dose in an infinitely old sample, and could provide correction for ages up to $325 \mathrm{ka}$. This contribution examines these fading correction procedures on basalt samples of known ages.

\section{SAMPLES AND EXPERIMENTAL DETAILS}

Eight volcanic samples were studied from Flores (FL) and Fayal (FA) Islands, Azores, Portugal. The rocks correspond to the basalt and hawaiite types in the age range of $400 \mathrm{ka}$ to $2.2 \mathrm{Ma}$. The existing chronology is based on volcano-stratigraphic methods and correlations with previous radiometric determinations on equivalent samples (see details in Azevedo and Portugal Ferreira, 2006). The details of these samples are summarized in Table 1. Samples for luminescence measurements were prepared after removing the outer layer of the collected 
Table 1. Tabulated values of measured $\mathrm{D}_{\mathrm{e}}$, dose rates and age estimates for 8 basaltic rock samples. Expected ages are also given.

\begin{tabular}{lcccc}
\hline Sample & $D_{e}(\mathbf{G y})$ & Dose rate (Gy-ka-1) & Age (ka) & Expected age $^{*}(\mathbf{k a})$ \\
\hline FL2 & $161 \pm 3$ & $2.6 \pm 0.1$ & $62.1 \pm 2.6$ & $1000-1500$ \\
\hline FL3 & $69.9 \pm 1.9$ & $3.0 \pm 0.1$ & $23.0 \pm 1.0$ & 400 \\
\hline FL4 & $149.2 \pm 3.7$ & $2.7 \pm 0.1$ & $55.3 \pm 2.5$ & $2000-2200$ \\
\hline FL5 & $88.9 \pm 1.2$ & $3.0 \pm 0.1$ & $29.6 \pm 1.2$ & $500-670$ \\
\hline FL6 & $68.2 \pm 1.2$ & $0.8 \pm 0.1$ & $85.2 \pm 4.5$ & 400 \\
\hline FL10 & $166.8 \pm 1.9$ & $3.2 \pm 0.1$ & $52.1 \pm 1.7$ & 670 \\
\hline FA2 & $56.5 \pm 2.3$ & $1.6 \pm 0.1$ & $35.3 \pm 2.6$ & $<1000$ \\
\hline FA5 & $1.7 \pm 0.7$ & $2.3 \pm 0.1$ & $0.7 \pm 0.3$ & $<1000$ \\
\hline
\end{tabular}

*Based on volcano-stratigraphic methods and correlations with previous radiometric determinations on equivalent samples (Azevedo and Portugal Ferreira, 2006).

rock pieces in the dark by sawing and thereafter crushing the inner material. The size fraction $150-210 \mu \mathrm{m}$ was then obtained by sieving and used for luminescence measurements without any further chemical treatment.

The light exposed part of the rock materials were crushed further to make it powder like. These powder samples were used to estimate the elemental concentration of $\mathrm{U}$, Th and $\mathrm{K}$ using gamma spectrometry with $\mathrm{NaI}(\mathrm{Tl})$ crystal detector. The alpha efficiency $(a)$ value of 0.1 was assumed in order to calculate the alpha dose contribution. Using the dose and dose rate values, the age estimates were derived and they are given in Table $\mathbf{1}$.

The measurements were carried out using a Ris $\varnothing$ TL/OSL-DA-15 reader. Blue light stimulation used an array of LEDs $(470 \pm 30 \mathrm{~nm})$ filtered through GG-420 long-pass filters, and delivering $\sim 50 \mathrm{~mW} \cdot \mathrm{cm}^{-2}$ at the sample position. IR stimulation was carried out using IR LEDs $(870 \pm 40 \mathrm{~nm})$ with a maximum intensity of $\sim 150 \mathrm{~mW} \cdot \mathrm{cm}^{-2}$. Calibrated beta sources $\left({ }^{90} \mathrm{Sr}{ }^{90} \mathrm{Y}\right)$ delivering between 0.22 (Risoe 1), 0.11 (Risoe 2) and 0.057 (PRL) Gy $\mathrm{s}^{-1}$ were used to irradiate the samples in the reader. An EMI 9635QB photomultiplier tube with bialkali photocathode was used. Blue emission $(320-460 \mathrm{~nm})$ was detected through a $4 \mathrm{~mm}$ Corning 7-59 and $2 \mathrm{~mm}$ Schott BG-39 optical filter combination. UV emission $(280-380 \mathrm{~nm})$ was detected through $7.5 \mathrm{~mm}$ of Hoya U-340 filter.

\section{MEASUREMENT PROTOCOLS}

The $D_{e}$ values were measured by single aliquot regenerative (SAR) procedure (Murray and Wintle, 2000) where a test dose induced luminescence signal was used to correct the possible sensitivity changes. The details about the preheat and the stimulation temperatures are given in the subsequent sections. The fading measurements were carried out using SAR procedure where the luminescence signals of prompt and various time delays were measured (Auclair et al., 2003). The fading rates ( $g$-values in \%/decade) were calculated by fitting Eq. 3.1 to the measured data of sensitivity corrected luminescence vs. time delay, $t_{d}$,

$$
I=I_{0}\left[1-g \log \left(\frac{t_{d}}{t_{c}}\right)\right]
$$

where $I$ and $I_{0}$ are the sensitivity corrected luminescence at $t_{d}$ (time delay) and $t_{c}$ (prompt) respectively. The $g$-values are standardized to $t_{c}=2$ days.

\section{SIGNAL SELECTION}

In the present sample conventional blue emission under IR stimulation at $50^{\circ} \mathrm{C}\left(\mathrm{OSL}_{\mathrm{IR}-\mathrm{B}}\left[50^{\circ} \mathrm{C}\right]\right)$, was close to the limits of detection and hence was not used. Then blue emission under IR stimulation at elevated temperature $\left(200^{\circ} \mathrm{C}\right)$ was measured (Tsukamoto et al., 2011). The decay curves of this signal of samples FL4 and FL5 are shown in Fig 1. The arithmetic mean of the measured $D_{e}$ values of FL4 and FL5 are $170 \pm 26$ Gy and $3 \pm 6$ Gy respectively. Using a test dose error $(20 \%)$ and recycling ratio (0.8-1.2), only 8 out of 12 aliquots were accepted in case of sample FL4. For FL5 the number of acceptable results was 6 out of 12 aliquots. The arithmetic means of $g$-values of FL4 and FL5 are $16 \pm 4$ and $17 \pm 2 \%$ /decade,

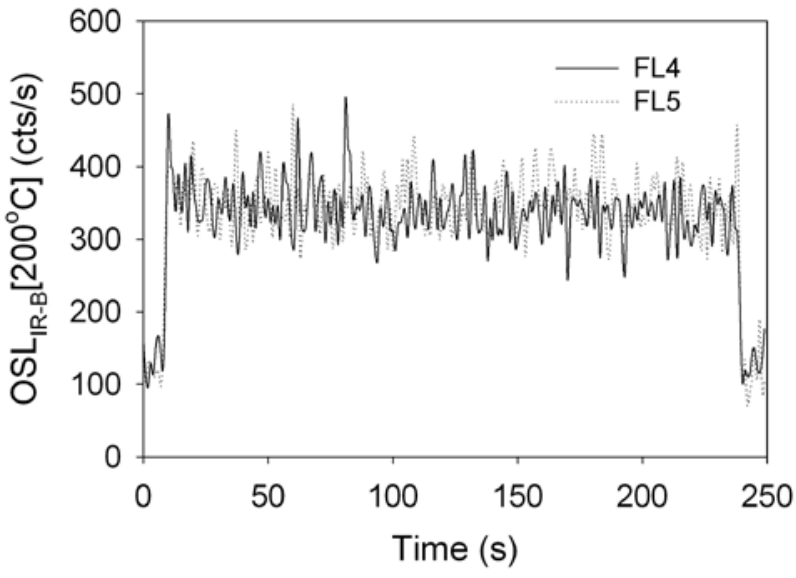

Fig. 1. OSLIR-B measured at $200^{\circ} \mathrm{C}$ is shown for two samples, a) FL4 and b) FL5. 
respectively. Because of low signal intensity and large $g$ values, the $\mathrm{OSL}_{\mathrm{IR}-\mathrm{B}}\left[200^{\circ} \mathrm{C}\right]$ was not used for age calculation.

We then investigated the $\mathrm{OSL}_{\mathrm{B}-\mathrm{UV}}\left[50^{\circ} \mathrm{C}\right]$ signal with a preheat of $250^{\circ} \mathrm{C}$ at $2^{\circ} \mathrm{C} / \mathrm{s}$ for $60 \mathrm{~s}$. The natural and $220 \mathrm{~Gy}$ beta dose induced luminescence signals of FL4 are shown in Fig. 2. The natural signal was undetectable and hence this signal was not considered for age calculation. Then the $\mathrm{OSL}_{\mathrm{B}-\mathrm{Uv}}\left[225^{\circ} \mathrm{C}\right]$ signal with a preheat of $250^{\circ} \mathrm{C}$ at $2^{\circ} \mathrm{C} / \mathrm{s}$ for $150 \mathrm{~s}$ was explored. The measured $D_{e}$ values of FL2, FL3, FL4, FL5, FL6 and FL10 are $22.2 \pm 0.4,8.1 \pm 0.4,25 \pm 1,13 \pm 1,7 \pm 1$ and $18 \pm 1$ Gy respectively. All the values are the arithmetic mean of 4 aliquots for each sample. The $D_{e}$ values of FA2 and FA5 were $11.5 \pm 0.4$ and $0.8 \pm 0.2$ Gy using 12 aliquots each. The arithmetic mean of the measured $g$-values is $23.5 \pm 1.3 \% /$ decade for all the 8 samples. The widely used correction procedure (Huntley and Lamothe, 2001) does not work for such high fading values (Morthekai et al., 2008) as its use results in negative intensity in a relatively shorter time scales and hence put a limit for age correction. Hence this signal was also not considered.

Earlier studies have shown a $\mathrm{TL}_{\mathrm{UV}}$ signal around $525^{\circ} \mathrm{C}$ in the basaltic materials (Guerin and Valladas, 1980) and that could be bleached by blue light (Morthekai et al., 2008). We explored this signal further. Some of its characteristics are mentioned elsewhere (Morthekai et al., 2008). It comprises two TL glow peaks at $525^{\circ} \mathrm{C}$ and $600^{\circ} \mathrm{C}$ (heating rate, $2^{\circ} \mathrm{C} / \mathrm{s}$ ) after an additional laboratory dose and a preheat of $380^{\circ} \mathrm{C}$ for $150 \mathrm{~s}$. The $525^{\circ} \mathrm{C}$ peak could be bleached by blue light but not the $600^{\circ} \mathrm{C}$ peak (Fig. 3). For the rest of this study, the OSL (blue stimulation) signal at $360^{\circ} \mathrm{C}, \mathrm{OSL}_{\mathrm{B}-\mathrm{Uv}}\left[360^{\circ} \mathrm{C}\right]$, was used in this study for age calculation.

\section{AGE CALCULATION}

The palaeodoses of each sample were measured using single aliquot regenerative (SAR) dose protocol (Murray

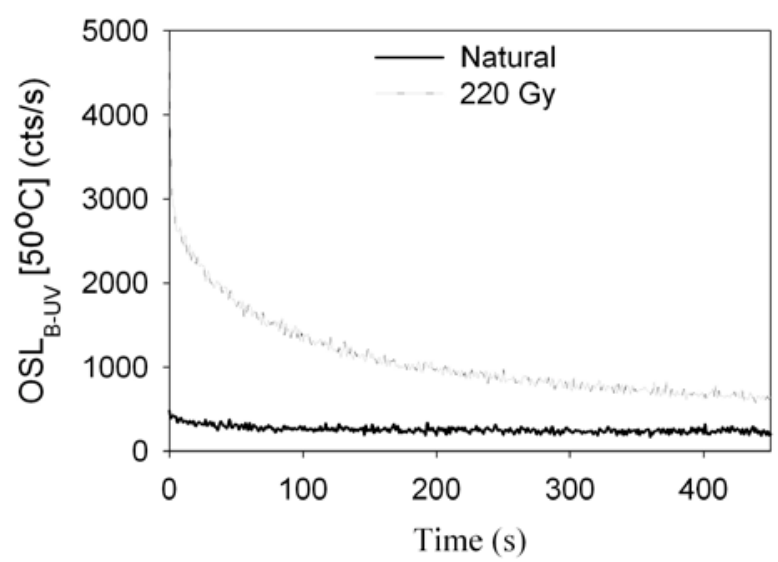

Fig. 2. OSLB-uv signals, both natural and laboratory irradiated, measured at $50^{\circ} \mathrm{C}$ temperature, are shown. and Wintle, 2000). Samples were preheated to $380^{\circ} \mathrm{C}$ at the heating rate $2^{\circ} \mathrm{C} / \mathrm{s}$ and kept for $150 \mathrm{~s}$ to reduce the ITL at the stimulation temperature i.e., $360^{\circ} \mathrm{C}$. Recycling ratio was in the range of 0.9-1.1 and the test dose error was within $10 \%$. The sensitivity corrected natural OSL signal was interpolated into the single saturated exponential dose response curve, DRC, to get the palaeodose. Twelve aliquots were measured for each sample. Using the dose and dose rate values, the age estimates were derived and they are given in Table 1. Except FA2 and FA5, the ages are only $10 \%$ of the expected ages and the underestimation might be because of anomalous fading in $\mathrm{OSL}_{\mathrm{B}-\mathrm{UV}}\left[360^{\circ} \mathrm{C}\right]$ also.

\section{FADING CORRECTION}

The fading of $\mathrm{OSL}_{\mathrm{B}-\mathrm{UV}}\left[360^{\circ} \mathrm{C}\right]$ signal was measured for all the 8 samples and the fading was corrected using two different procedures $(\mathrm{CP})$ :

CP1: The correction procedure of Huntley and Lamothe, 2001 and

CP2: The correction procedure of Kars et al., 2008 and Kars and Wallinga, 2009.

$\mathrm{CP} 1$ is based on the understanding that the anomalous fading follows logarithmic decay with time while CP2 assumed that it depends on the number density of luminescence centres and the fading follows $\exp \left[-\rho^{\prime} \ln (s t)^{3}\right]$ with time where $\rho^{\prime}$ is the fading parameter related to the number density of luminescence centres in the crystal and $s$ is the attempt-to-escape frequency factor in $\mathrm{s}^{-1}$ (Huntley, 2006). The details of the procedures are given in the subsequent sections.

\section{Correction procedure CP1}

This is widely used procedure and is based on three assumptions, viz.,

1) trapped charges are received by the crystal at constant rate due to irradiation,

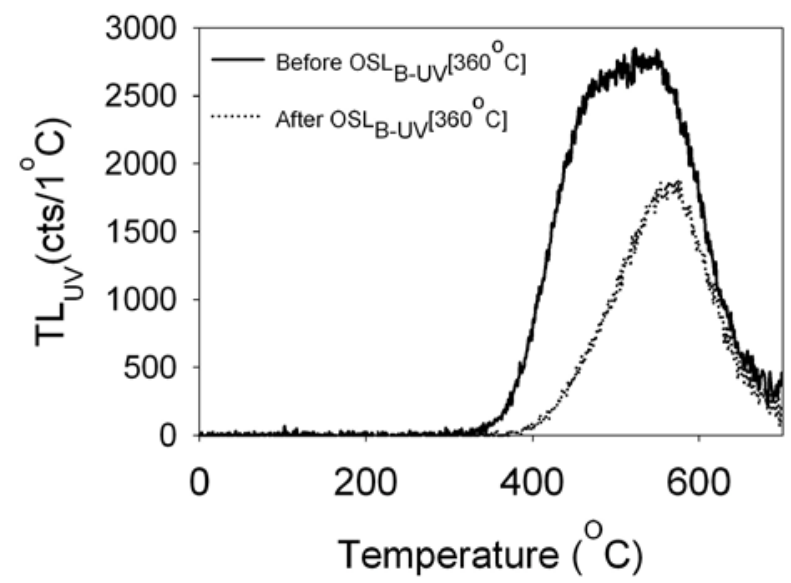

Fig. 3. Thermoluminescence signals peak at $525^{\circ} \mathrm{C}$ and $600^{\circ} \mathrm{C}$ at $2^{\circ} \mathrm{C} / \mathrm{s}$ after an additional laboratory dose and a preheat of $360^{\circ} \mathrm{C}$ for $150 \mathrm{~s}$ before and after blue light stimulation for $500 \mathrm{~s}$. 
2) every increment of trapped charges recombine independent of earlier increments and

3) luminescence signal fades logarithmically with time.

The implication of the first and second assumptions is that the natural luminescence lies in the linear part of the DRC. The third assumption predicts negative luminescence intensity after a certain time since the cessation of irradiation and limits the correction beyond that time.

With the exception of FL4, the condition based on first and second assumptions was met in all samples. The measured dose responses and the natural sensitivity corrected luminescence signal, $L / T$ for FL2, FL4 and FL10 are shown in Fig. 4.

In $\mathrm{CP} 1$, as per the third assumption, the sensitivity corrected luminescence signals $(L / T)$ is plotted against $\log \left[t_{d}\right]$ where $t_{d}$ is delay time since the cessation of laboratory irradiation which is roughly the sum of half of the irradiation time and time taken to preheat (Auclair et al., 2003). Then the data is fitted with Eq. 3.1 (Fig. 5) and the fading parameter, $g$, is derived from the slope.

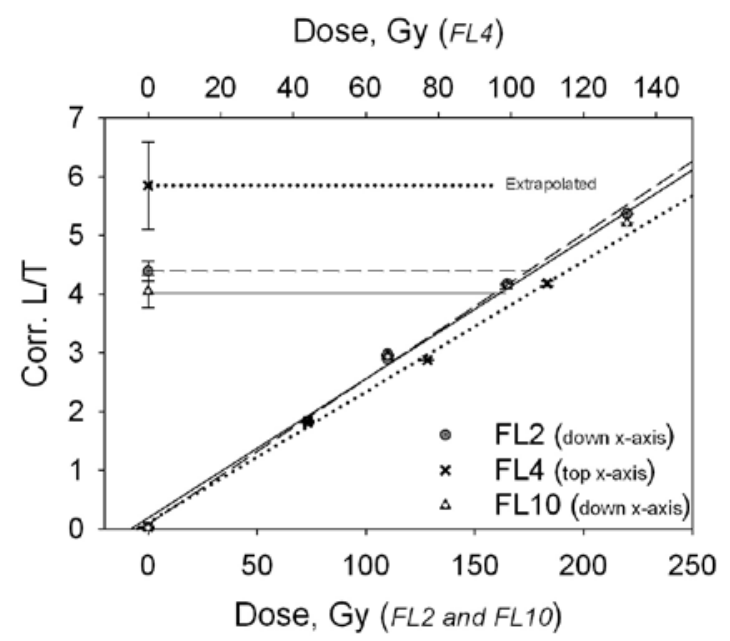

Fig. 4. Measured dose response curves of FL2, FL4 and FL10. Except FL4, natural $\mathrm{L} / \mathrm{T}$ points are well bracketed by the regenerative dose points.
The correction formula (Eq. 6.1) which is the integration of the fading signals throughout the irradiation by assuming each increment of trapped charges is decaying independent of each other and the linearity between $I$ and $t$ with the rate $I_{0} / T$ (first and second assumptions) is given as

$T_{f}=T\left[1-k\left\{\ln \left(\frac{T}{t_{c}}\right)-1\right\}\right]$

where $T_{f}$ and $T$ are the measured and corrected ages and $k=g /(100 \cdot \ln [10])$ (Aitken, 1985, Huntley and Lamothe, 2001). Thus obtained ages are given in Table 2. The corrected ages are in gross under-estimation of $80 \%$ even though the conditions to apply this procedure are met.

\section{Correction procedure CP2}

This procedure by Kars et al. (2008) and Kars and Wallinga (2009) is based on Huntley (2006). The fading parameter, $\rho$ ' is obtained from the Eq. $\mathbf{6 . 2}$ by fitting to the fading measurements. One such fitting for the sample FL4 is given in Fig. 6 (thick line).

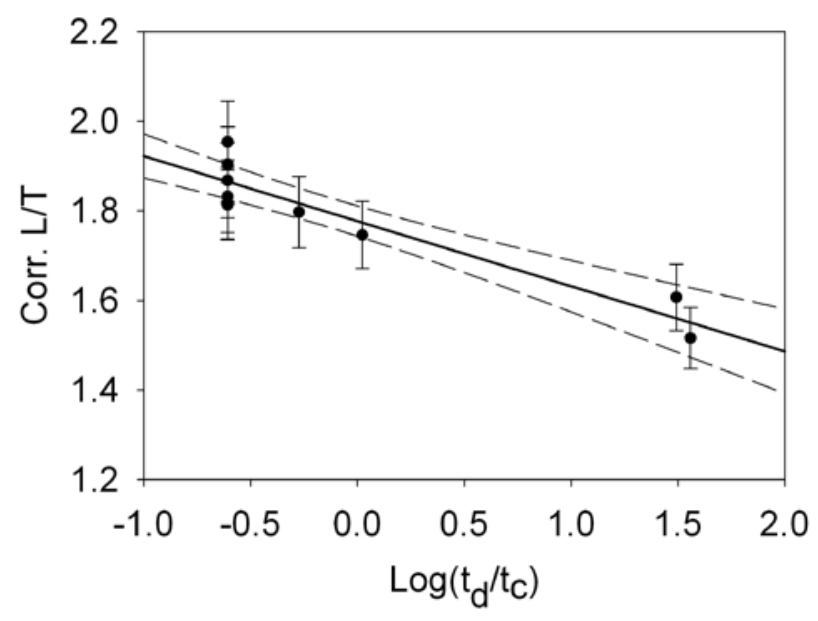

Fig. 5. Fading measurements of FL4 and fitting of Eq. 3.1 to the data and $\mathrm{t}_{\mathrm{c}}$ is taken to be 1 hour.

Table 2. Tabulated values of uncorrected and corrected age estimates by CP1 for all the samples. Fading parameter, g-value is standardized to $t_{c}=2$ days.

\begin{tabular}{lccccc}
\hline Sample & $\begin{array}{c}\text { Apparent age } \\
(\mathbf{k a})\end{array}$ & $\begin{array}{c}\text { g-value } \\
(\% / \mathrm{dec})\end{array}$ & $\begin{array}{c}\text { Delay time* } \\
\text { (days) }\end{array}$ & $\begin{array}{c}\text { Corrected agecp1 } \\
\text { (ka) }\end{array}$ & $\begin{array}{c}\text { Expected age } \\
\text { (ka) }\end{array}$ \\
\hline FL2 & $62.1 \pm 2.6$ & $10.2 \pm 0.7$ & 6 & $219.8 \pm 12.5$ & $1000-1500$ \\
\hline FL3 & $23.0 \pm 1.0$ & $8.1 \pm 0.9$ & 6 & $46.7 \pm 3.4$ & 400 \\
\hline FL4 & $55.3 \pm 2.5$ & $9.5 \pm 0.8$ & 84 & $138.4 \pm 9.4$ & $2000-2200$ \\
\hline FL5 & $29.6 \pm 1.2$ & $9.3 \pm 0.5$ & 84 & $65.8 \pm 4.1$ & $500-670$ \\
\hline FL6 & $85.2 \pm 4.5$ & $7.7 \pm 0.8$ & 5 & $181.8 \pm 16.3$ & 400 \\
\hline FL10 & $52.1 \pm 1.7$ & $8.4 \pm 0.6$ & 5 & $119.9 \pm 6.2$ & 670 \\
\hline FA2 & $35.3 \pm 2.6$ & $9.8 \pm 2.7$ & 12 & $98.3 \pm 11.3$ & $<1000$ \\
\hline FA5 & $0.7 \pm 0.3$ & $11.4 \pm 0.9$ & 14 & $1.5 \pm 1.2$ & $<1000$ \\
\hline
\end{tabular}

tc used in Eq. 6.1. 


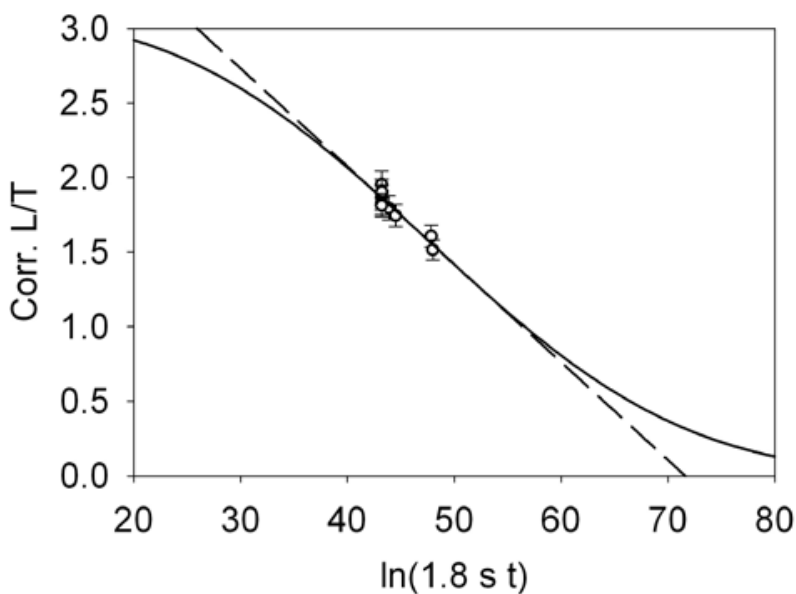

Fig. 6. Obtaining fading parameters ( $\mathrm{g}$ and $\rho^{\prime}$ ) by fitting Eq. 3.1 (as in Fig. 5) and Eq. 6.3 to the fading measurements of FL4. Both functions fit very well in that limited measurable time scales in the laboratory.

$$
I=I_{0} \mathrm{e}^{-\rho^{\prime} \ln (1.8 s t)^{3}}
$$

where $I$ and $I_{0}$ are the intensity of luminescence signals at time $\mathrm{t}$ and at immediately after the irradiation and $s$ is the attempt to escape factor assumed to be $3.0 \cdot 10^{15} \mathrm{~s}^{-1}$. The correction is done as follows,

1) the natural OSL signal and the dose response curve with three or more regenerative points are measured

2) each of regenerative OSL points are corrected for the fading during the laboratory irradiation using Eq. 6.2 with $t(\mathrm{~s})$ as $0.5 \cdot t_{R}+550$ where $\mathrm{t}_{\mathrm{R}}$ is the regeneration beta dose exposure time and $550 \mathrm{~s}$ is the time delay from irradiation to prompt measurement.

3) this corrected dose response curve is fitted to a single saturating exponential to get the saturating limit $I_{0}$ of the sensitivity corrected OSL and $D_{0}$, the onset of saturation

4) construct a dose response curve with a natural dose rate assuming $I_{0}$ is the limit for the OSL and the corresponding trapped charges are distributed in the crystal with the distance distribution with respect to the recombination centres as described by Eq. 6.3 given below $p\left(r^{\prime}\right) d r^{\prime}=3 r^{\prime^{2}} \mathrm{e}^{-r^{\prime}} d r^{\prime}$

where $r^{\prime}$ is the dimensionless distance variable (Eq. 3 of Huntley, 2006). The $r_{a v}^{\prime}$ and $r_{\max }^{\prime}$ are calculated to be 0.893 and 0.874 respectively (Fig. 10a) and the derivations are given in Appendix. The issues of fading during irradiation and the evolution of different types of traps ( 250 types in this case) throughout the natural irradiation were taken care of (see Kars and Wallinga, 2009 for more details).

5) the measured natural OSL is to be interpolated into this constructed dose response curve which mimics the same during natural irradiation.

The fading parameter, $\rho^{\prime}$, of all the samples were derived and the arithmetic mean and standard deviation is $(5.63 \pm 0.83) \cdot 10^{-6}$. As it is required to have full dose response curve for the calculation, that of FL4 was measured with the intention to use it as a standardized response curve. The standardized curves of 4 samples are shown in Fig. 7 and all the L/T measurements were corrected for the fading that occurred during the test dose irradiation like the step 2 above (except the $t_{R}$ is replaced by $t_{T D}$, time to give test dose). The measured full response

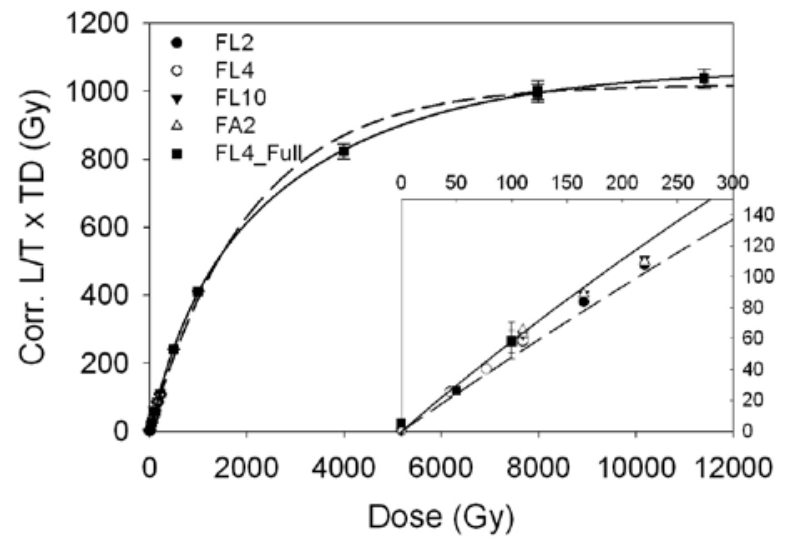

Fig. 7. Standardized dose response curves of the FL2, FL4, FL10 and FA2 and one full dose response curve of FL4. They are fitted with single (dashed line) and double (thick line) saturating exponentials. Inset shows the form of the dose response curve is same for all the samples.

Table 3. Tabulation of uncorrected and corrected age estimates by CP2.

\begin{tabular}{lcccc}
\hline Sample & Apparent age (ka) & Corrected agecP2 (ka) & Expected age (ka) & Under-estimation (\%) \\
\hline FL2 & $62.1 \pm 2.6$ & $407 \pm 22$ & $1000-1500$ & 65 \\
\hline FL3 & $23.0 \pm 1.0$ & $168 \pm 15$ & 400 & 54 \\
\hline FL4 & $55.3 \pm 2.5$ & $350 \pm 57$ & $2000-2200$ & 80 \\
\hline FL5 & $29.6 \pm 1.2$ & $193 \pm 6$ & $500-670$ & 64 \\
\hline FL6 & $85.2 \pm 4.5$ & $157 \pm 46$ & 400 & 49 \\
\hline FL10 & $52.1 \pm 1.7$ & $362 \pm 33$ & 670 & 38 \\
\hline FA2 & $35.3 \pm 2.6$ & $124 \pm 8$ & $<1000$ & $<73$ \\
\hline FA5 & $0.7 \pm 0.3$ & $5 \pm 1$ & $<1000$ & $<98$ \\
\hline
\end{tabular}


of FL4 has been used for further calculations. The natural $L / T$ points for all the samples were also corrected for test dose and fading during test dose irradiation. The test dose correction was necessary because the test dose for natural $L / T$ and the regeneration $L / T \mathrm{~s}$ were different. The test dose for natural $L / T \mathrm{~s}$ was 33 Gy (for FL4 and FL5, that was $22 \mathrm{~Gy}$ ) and that for regeneration $L / T \mathrm{~s}$ was $85.3 \mathrm{~Gy}$. This standard response curve was corrected for fading during regeneration doses, as mentioned in step 2, using average $\rho^{\prime}$. Using that the natural dose response curve was constructed and here also the average $\rho^{\prime}$ was used. Dose estimates were made by interpolating the corrected $L / T$ points into the constructed standardized natural dose response curve. The measured dose response curve, unfaded dose response curve and the constructed natural dose response curves along with the highest natural $L / T$ are shown in Fig. 8 and the computed ages are given in Table 3. The associated error with the corrected ages is the projection of standard deviation of $L / T$ of 6 to 12 aliquots of each sample into the constructed DRC.

\section{DISCUSSION}

The CP1 grossly underestimates the ages and yields only $20 \%$ compared to the expected ages. In our samples, even first and second assumptions are satisfied third assumption would not have been satisfied. Third assumption will be invalid if the sample is too old and the fading rates are high (Huntley and Lamothe, 2001). If the sample is too old and for higher fading rates, the fading scheme can not be approximated with the logarithmic decay for the whole burial period. Hence the fading correction by extrapolating the fading of laboratory induced signals to the burial period in million years would give, most probably, an underestimated age. The failure of third assumption could be the reason for the huge underestimation of ages by $\mathrm{CP} 1$.

The CP2 corrected ages were also underestimated and yielded only $40 \%$ compared to the expected ages. Assumed values for $s, \alpha$ and barrier height are $3 \cdot 10^{15} \mathrm{~s}^{-1}$, $9 \cdot 10^{9} \mathrm{~m}^{-1}$ and $3 \mathrm{eV}$ respectively and they are related to $\mathrm{OSL}_{\mathrm{IR}-\mathrm{B}}$ signal from feldspar. For the $\mathrm{OSL}_{\mathrm{B}-\mathrm{UV}}\left[360^{\circ} \mathrm{C}\right]$ signal, used in this study, the $s$ value is not available. If we change $s$ value to $10^{5} \mathrm{~s}^{-1}$ also remove the factor 1.8 from the fading equation (Eq. 6.2), then the obtained arithmetic mean of $\rho^{\prime}$ is $(26.5 \pm 0.4) \cdot 10^{-6}$ (range: $21.0 \cdot 10^{-6}$ to $\left.33.2 \cdot 10^{-6}\right)$. If one reconstructs natural DRC using maximum value of $\rho 33.2 \cdot 10^{-6}$ then the corrected ages are consistent with the expected ages for 5 out of 8 samples (Table 4). The constructed DRCs using both the $s\left(3 \cdot 10^{15}\right.$ and $\left.10^{5} \mathrm{~s}^{-1}\right)$ are given in Fig. 9. The corresponding nearest neighbour luminescence centre distribution at various times in nature are plotted in Fig. 10 for a) $s=$ $3 \cdot 10^{15}$ and b) $10^{5} \mathrm{~s}^{-1}$ respectively.

It can be noticed that only a small fraction of the signals is stable using which the natural DRC's are constructed (Fig. 9). The regenerated signals constructed using the latter value is more stable as compared to the former. This might partly account for the age underestimation. These ages are encouraging and require further investigations.

\section{SUMMARY}

$\mathrm{OSL}_{\text {IR-B }}\left[50^{\circ} \mathrm{C}\right], \mathrm{OSL}_{\text {IR-B }}\left[200^{\circ} \mathrm{C}\right], \mathrm{OSL}_{\mathrm{B}-\mathrm{UV}}\left[50^{\circ} \mathrm{C}\right]$ and $\mathrm{OSL}_{\mathrm{B}-\mathrm{UV}}\left[225^{\circ} \mathrm{C}\right]$ are found that these signals can not be used to estimate the ages for the volcanic samples studied

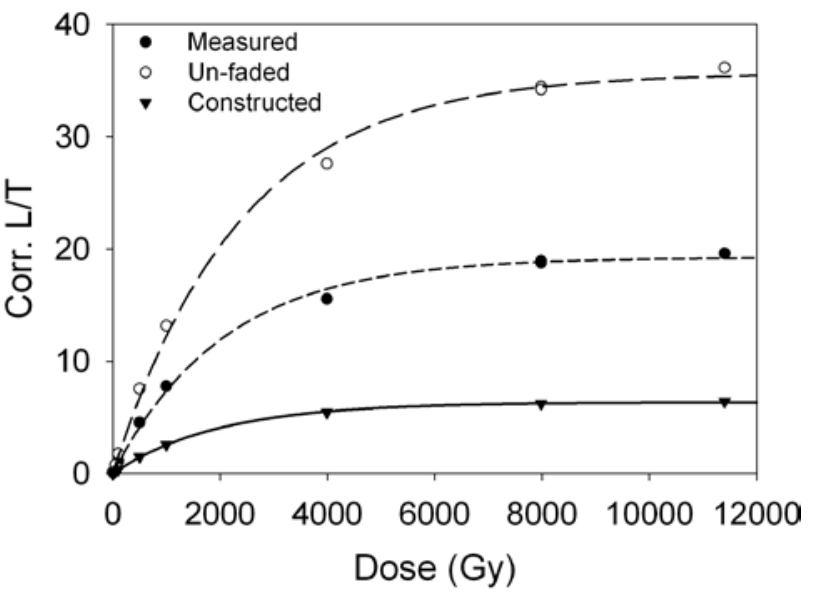

Fig. 8. The measured dose response curve, unfaded dose response curve and the constructed natural dose response curves are shown. The highest natural $\mathrm{L} / \mathrm{T}$ point is also shown.

Table 4. Comparison of corrected ages by CP2 for two values of attempt-to-escape frequency factor, s. '-' sign indicates overestimation.

\begin{tabular}{|c|c|c|c|c|}
\hline Sample & $\begin{array}{c}\text { Corrected age } \\
(\mathrm{ka})\end{array}$ & $\begin{array}{c}\text { Corrected age } e_{C P 2} \text { for } s=10^{5} \mathrm{~s}^{-1} \\
\text { (ka) }\end{array}$ & $\begin{array}{l}\text { Expected age } \\
\text { (ka) }\end{array}$ & $\begin{array}{c}\text { Under or over estimation } \\
(\%)\end{array}$ \\
\hline$\overline{F L 2}$ & $407 \pm 22$ & $1139 \pm 140$ & $1000-1500$ & -2 \\
\hline FL3 & $168 \pm 15$ & $305 \pm 32$ & 400 & 15 \\
\hline FL4 & $350 \pm 57$ & $835 \pm 248$ & $2000-2200$ & 48 \\
\hline FL5 & $193 \pm 6$ & $360 \pm 13$ & $500-670$ & 34 \\
\hline FL6 & $157 \pm 46$ & $324 \pm 116$ & 400 & -10 \\
\hline FL10 & $362 \pm 33$ & $856 \pm 138$ & 670 & -7 \\
\hline FA2 & $124 \pm 8$ & $228 \pm 16$ & $<1000$ & 51 \\
\hline FA5 & $5 \pm 1$ & $8 \pm 2$ & $<1000$ & 98 \\
\hline
\end{tabular}




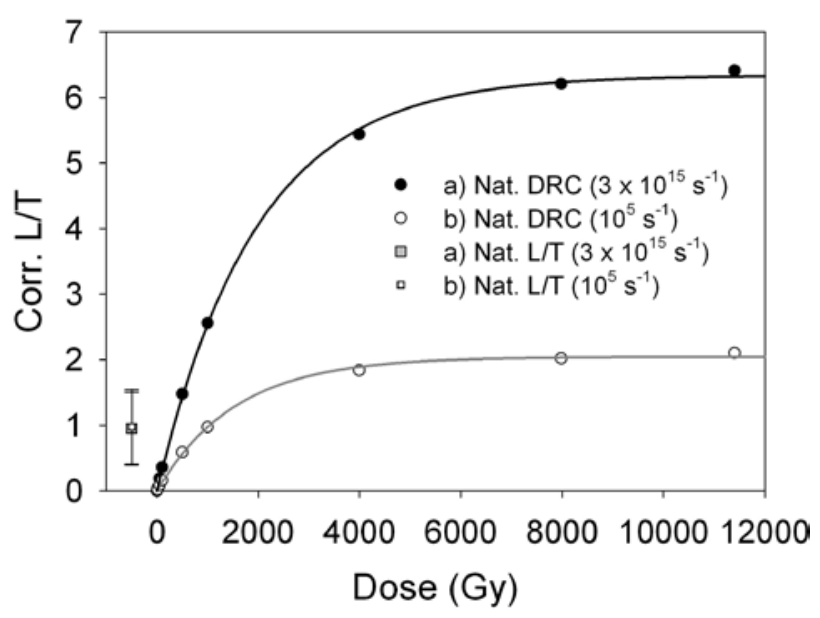

Fig. 9. The constructed natural DRCs using attempt-to-escape frequency factor, s, a) $3 \cdot 10^{15} \mathrm{~s}^{-1}$ and b) $10^{5} \mathrm{~s}^{-1}$. For clarity the natural $\mathrm{L} / \mathrm{T}$ points are plotted earlier in the Dose axis.
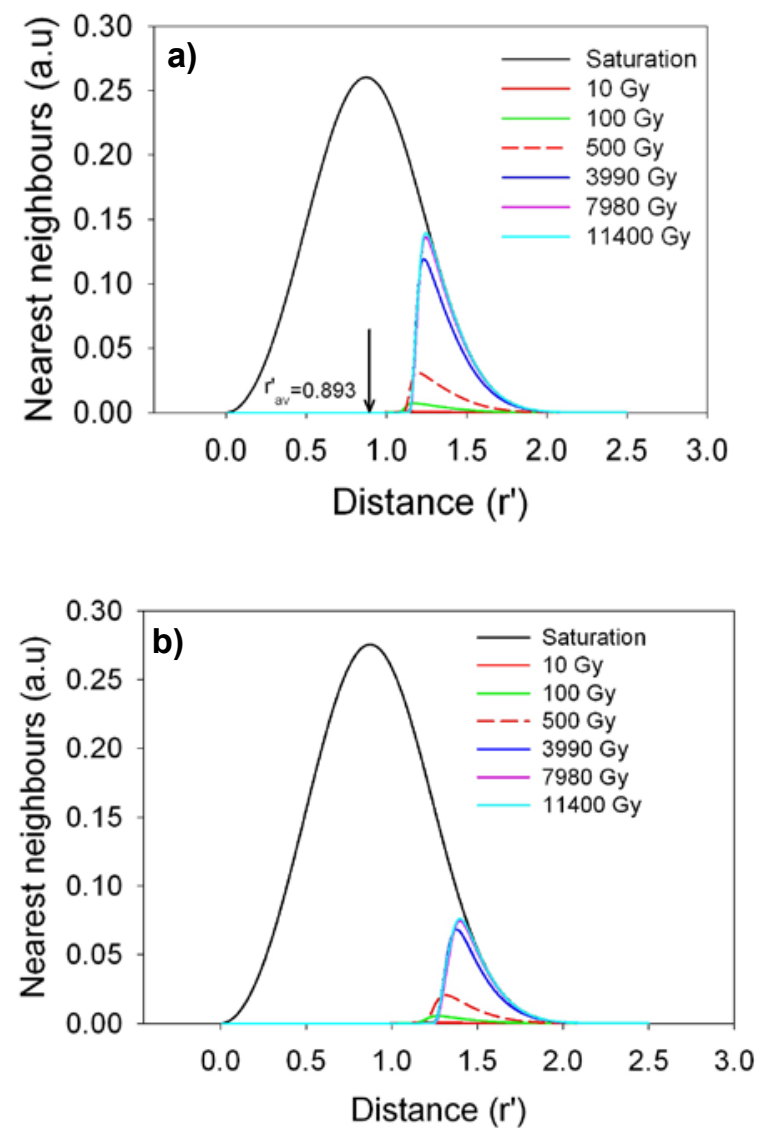

Fig. 10. The nearest neighbour hole distribution at various times (doses for a constant dose rate) in nature obtained using $\mathrm{s}$, a) $3 \cdot 10^{15} \mathrm{~s}^{-1}$ and b) $10^{5} \mathrm{~s}^{-1}$. The abscissa is the dimensionless distance variable, $r^{\prime}$ as given in Eq. 6.3. Stable signals commences from $r^{\prime}$ a) $\sim 1.03$ and b) $\sim 1.20$ and $r_{a v}^{\prime}$ is indicated for comparison. here. $\mathrm{OSL}_{\mathrm{B}-\mathrm{UV}}\left[360^{\circ} \mathrm{C}\right]$ signal was used to date the volcanic eruption event of 8 basaltic rock samples. The measured ages were underestimated compared to the expected ages and the underestimation was attributed to anomalous fading. We applied two procedures 1) Huntley and Lamothe, 2001, and 2) Kars et al., 2008 for fading correction. Using the latter correction procedure, the corrected ages have a deviation of more than $60 \%$ from the expected ages. However, if the $s$ value is changed from $3 \cdot 10^{15} \mathrm{~s}^{-1}$ to $10^{5} \mathrm{~s}^{-1}$, then the corrected ages have a better agreement with the expected ages. This is an interesting result worth further investigations although we acknowledge that our $s$ value is unrealistically small.

\section{ACKNOWLEDGEMENTS}

R. H. Kars is acknowledged for providing the data set to verify our spreadsheet. K. D. Ansari and R. H. Biswas are acknowledged for their inputs.

\section{REFERENCES}

Aitken MJ, 1985. Thermoluminescence dating. Academic Press, London: $359 \mathrm{pp}$

Auclair M, Lamothe M and Huot S, 2003. Measurement of anomalous fading for feldpsar IRSL using SAR. Radiation Measurements 37(4-5): 487-492, DOI 10.1016/S1350-4487(03)00018-0.

Azevedo JMM and Portugal Ferreira MR, 2006. The volcano-techtonic evolution of Flores Island, Azores (Portugal). Journal of Volcanology and Geothermal Research 156(1-2): 90-112, DOI 10.1016/j.jvolgeores.2006.03.011.

Chandrasekhar S, 1943. Stochastic Problems in Physics and Astronomy. Reviews of Modern Physics 15(1): 86-87.

Guerin G and Valladas G, 1980. Thermoluminescence dating of volcanic plagioclases. Nature 286(5774): 697-699, DOI 10.1038/286697a0.

Huntley DJ, 2006. An explanation of the power-law decay of luminescence. Journal of Physics: Condensed Matter 18(4): 1359-1365, DOI 10.1088/0953-8984/18/4/020.

Huntley DJ and Lamothe M, 2001. Ubiquity of anomalous fading in Kfeldspars and the measurement and correction for it in optical dating. Canadian Journal of Earth Sciences 38(7): 1093-1106, DOI 10.1139/e01-013.

Kars RH and Wallinga J, 2009. IRSL dating of K-feldspars: Modelling natural dose response curves to deal with anomalous fading and trap competition. Radiation Measurements 44(5-6): 594-599, DOI 10.1016/j.radmeas.2009.03.032.

Kars RH, Wallinga $\mathrm{J}$ and Cohen KM, 2008. A new approach towards anomalous fading correction for feldspar IRSL dating - tests on samples in field saturation. Radiation Measurements 43(2-6): 786790, DOI 10.1016/j.radmeas.2008.01.021.

Morthekai P, Jain M, Murray AS, Thomsen KJ and Bøtter-Jensen L, 2008. Fading characteristics of Martian analogue materials and the applicability of a correction procedure. Radiation Measurements 43(2-6): 672-678, DOI 10.1016/j.radmeas.2008.02.0.

Murray AS and Wintle A, 2000. Luminescence dating of quartz using an improved single-aliquot regenerative-dose protocol. Radiation Measurements 32(1): 57-73, DOI 10.1016/S1350-4487(99)00253$\mathrm{X}$.

Tsukamoto S, Duller GAT, Wintle A and Muhs D, 2011. Assessing the potential for luminescence dating of basalts. Quaternary Geochronology 6(1): 61-70, DOI 10.1016/j.quageo.2010.04.002. 


\section{APPENDIX}

The nearest neighbour distribution function, $p\left(r^{\prime}\right)$ is shown as below (Huntley, 2006),

$$
p\left(r^{\prime}\right) d r^{\prime}=3 r^{\prime 2} \mathrm{e}^{-r^{\prime 3}} d r^{\prime}
$$

where

$r^{\prime}=\left(\frac{4 \pi \rho}{3}\right)^{1 / 3} r$

and $\rho$ is the number density of luminescence centers $\left(\mathrm{m}^{-3}\right)$. And $\rho$ itself is related to $\rho^{\prime}$ via

$$
\rho^{\prime}=\left(\frac{4 \pi}{3 \alpha^{3}}\right) \rho
$$

where $\alpha$ is assumed to be $9 \cdot 10^{9} \mathrm{~m}^{-1}$ and this corresponds to barrier height of $3 \mathrm{eV}$.

The average $r^{\prime}$ is calculated as below (Chandrasekhar, 1943),

$r_{a v}^{\prime}=\int_{0}^{\infty} r^{\prime} p\left(r^{\prime}\right) d r^{\prime}$
Taking $t=r^{\prime 3} ; d t=3 r^{\prime 2} d r^{\prime}$, and after substituting these in Eq. A.4,

$$
r_{a v}^{\prime}=\int_{0}^{\infty} t \mathrm{e}^{-t} \frac{d t}{t^{2 / 3}}=\Gamma\left(\frac{4}{3}\right)=0.893
$$

The $r_{\max }^{\prime}$ is calculated by equating the first derivative of $p\left(r^{\prime}\right)$ to zero as below,

$$
\frac{d p\left(r^{\prime}\right)}{d r^{\prime}}=r^{\prime} e^{-r^{\prime 3}}\left(6-9 r^{\prime 3}\right)=0
$$

$r_{\text {max }}^{\prime}=\left(\frac{2}{3}\right)^{1 / 3}=0.874$.

Using Eqs. A.2 and A.5, $r_{a v}$ is calculated as 0.55407 $\rho^{-1 / 3}$ and $r_{\max }$ is calculated to be $0.54202 \rho^{-1 / 3}$ using Eq. A.2 and A.7. For a given $r$ value, the life time can be calculated using Eq. A.8,

$\tau_{a v}=s^{-1} e^{\alpha r_{a v}}$

For example, $\rho^{\prime}=3 \cdot 10^{-6}, r_{a v}=6.88 \mathrm{~nm}$ and the corresponding average life time is $8.21 \mathrm{ka}$. For $\rho^{\prime}=4.94 \cdot 10^{-6}$, $r_{a v}=5.93 \mathrm{~nm}$ and the corresponding average life time is 0.625 years. 